\title{
PROBLEM 26 OF L. FUCHS
}

\author{
CHIN-SHUI HSÜ
}

\begin{abstract}
This solves the following problem: Which Abelian groups are the inverse limits of Abelian groups, each of which is a finite direct sum of quasi-cyclic and bounded Abelian groups? (Here quasi-cyclic means isomorphic to some $Z\left(p^{\infty}\right)$.) A necessary and sufficient condition for an Abelian group to be such is that it takes the form $A_{r} \oplus \Pi_{p} \operatorname{Hom}_{z}\left(A_{p}, Z\left(p^{\infty}\right)\right)$ where $A_{r}$ is complete and reduced, the $A_{p}$ are torsion-free and the direct product is taken over the set of prime numbers.
\end{abstract}

We are going to solve the following problem of L. Fuchs [1]: Which Abelian groups are the inverse limits of Abelian groups each of which is a finite direct sum of quasi-cyclic and bounded Abelian groups?

We shall adopt the following notations for an Abelian group $A: A_{d}$ is its maximal divisible subgroup; $A_{r}=A \mid A_{d} ; A[n]=\{x \in A \mid n x=0\} ; T_{p}(A)$ is the p-primary component of the torsion subgroup of $A$. We let $Z$ denote the group of integers, $Q$ the rational numbers, $Z_{p}$ the $p$-adic integers, and $Z\left(p^{\infty}\right)=T_{p}(Q / Z)$. An Abelian group is quasi-cyclic if it is isomorphic to $Z\left(p^{\infty}\right)$ for some prime number $p$. To say that $A$ is a finite direct sum of quasi-cyclic and bounded Abelian groups is equivalent to the conditions: (a) $A_{d}$ is a finite direct sum of quasi-cyclic groups; (b) $A_{r}$ is bounded.

Let $R$ be a ring. An $R$-module shall mean a left $R$-module. A topology on an $R$-module $A$ shall be one in which the additive group of $A$ becomes a (Hausdorff) topological group. It is linear if there is an open base at 0 consisting of $R$-submodules. A linear topology on $A$ is linearly compact if it satisfies the condition: Given a family $\left\{K_{\omega}\right\}_{\omega \in \Omega}$ of residue classes of $A$ modulo closed $R$-submodules, if every finite subfamily has a nonempty intersection then $\bigcap_{\omega \in \Omega} K_{\omega} \neq \varnothing$.

Suppose that $A$ is an $R$-module and $A^{\prime}$ is an $R$-submodule with some topology. For $x \in A$, we call a subset of $x+A^{\prime}$ a linear subset if it has the form $y+B$, where $y \in x+A^{\prime}$ and $B$ is a closed $R$-submodule of $A$. Evidently we have

Leman 1. Let $A, B$ be $R$-modules, $A^{\prime}, B^{\prime}$ be respectively their submodules with some topologies, and $\varphi: A \rightarrow B$ be an $R$-homomorphism which induces a continuous $R$-homomorphism $A^{\prime} \rightarrow B^{\prime}$.

Received by the editors November 16, 1970 and, in revised form, November 22, 1971. AMS (MOS) subject classifications (1970). Primary 20K25. 
(i) If $\left\{K_{\omega}\right\}_{\omega \in \Omega}$ is a family of linear subsets of $x+A^{\prime}(x \in A)$, then $\bigcap_{\omega \in \Omega} K_{\omega}$ is either a linear subset or $\varnothing$.

Furthermore, if $A^{\prime}$ is either linearly compact or compact and every finite subfamily of $\left\{K_{\omega}\right\}_{\omega \in \Omega}$ has nonempty intersection then $\bigcap_{\omega \in \Omega} K_{\omega} \neq \varnothing$.

(ii) For $y \in \varphi(x)+B^{\prime}, \varphi^{-1}(y) \cap\left(x+A^{\prime}\right)$ is a linear subset of $x+A^{\prime}$.

(iii) If $A^{\prime}$ is either linearly compact or compact and $K$ is a linear subset of $x+A^{\prime}(x \in A)$, then $\varphi(K)$ is a linear subset of $\varphi(x)+B^{\prime}$.

The next lemma is due to C. U. Jensen [2]. An alternative proof is included here because it is more elementary and needs fewer assumptions than that of [2]. The idea of this proof is derived from [3, Proposition 13-2-1, p. 66]. (We were not aware of the result of [2] until Professor Joseph Rotman kindly informed us. We are also indebted to the referee for some improvements.)

LEMMA 2 (C. U. JENSEN). Let

$$
0 \longrightarrow\left\{A_{\alpha}^{\prime}, \pi_{\alpha \beta}^{\prime}\right\} \stackrel{\left\{\sigma_{\alpha}\right\}}{\longrightarrow}\left\{A_{\alpha}, \pi_{\alpha \beta}\right\} \stackrel{\left\{\tau_{\alpha}\right\}}{\longrightarrow}\left\{A_{\alpha}^{\prime \prime}, \pi_{\alpha \beta}^{\prime \prime}\right\} \longrightarrow 0
$$

be an exact sequence of inverse systems of $R$-modules where $A_{\alpha}^{\prime}$ are linearly compact (in some linear topologies) and $\pi_{\alpha \beta}^{\prime}$ are continuous, then $\tau=$ proj $\lim \tau_{\alpha}$ is onto.

The same conclusion also holds if $A_{\alpha}^{\prime}$ are compact (in some topologies) instead of linearly compact.

ProOf. Given $x=\left\{x_{\alpha}\right\} \in \operatorname{proj} \lim A_{\alpha}^{\prime \prime}$ we have an inverse system of sets $\left\{E_{\alpha}, f_{\alpha \beta}\right\}$, where $E_{\alpha}=\tau_{\alpha}^{-1}\left(x_{\alpha}\right)$ and $f_{\alpha \beta}: E_{\beta} \rightarrow E_{\alpha}$ are induced by $\pi_{\alpha \beta}$. By Lemma 1 , the conditions (i)-(iv) of [4, Theorem 1, p. 199] are satisfied (here $\mathfrak{S}_{\alpha}$ is the family of all linear subsets of $E_{\alpha}$ together with $\varnothing$ ). Therefore proj $\lim E_{\alpha}$ is nonempty. Let $z \in$ proj $\lim E_{\alpha}$, then we have $\tau(x)=x$, i.e., $\tau$ is onto.

REMARK. This proof also works for inverse systems of rings as well as (noncommutative) groups.

COROLlaRY 1. If $\left\{A_{\alpha}, \pi_{\alpha \beta}\right\}$ is an inverse system of divisible Abelian groups satisfying the conditions: For every positive integer $n$, (a) each $A_{\alpha}[n]$ has a compact topology, (b) each $\pi_{\alpha \beta}$ induces a continuous homomorphism $\pi_{\alpha \beta}[n]: A_{\beta}[n] \rightarrow A_{\alpha}[n]$, then proj $\lim A_{\alpha}$ is also divisible.

Proof. Given a positive integer $n$, we have an exact sequence

$$
0 \longrightarrow\left\{A_{\alpha}[n], \pi_{\alpha \beta}[n]\right\} \longrightarrow\left\{A_{\alpha}, \pi_{\alpha \beta}\right\} \stackrel{\left\{\tau_{\alpha}\right\}}{\longrightarrow}\left\{A_{\alpha}, \pi_{\alpha \beta}\right\} \longrightarrow 0
$$

where $\tau_{\alpha}(x)=n x$ for all $x \in A_{\alpha}$. By Lemma 2,

$$
\tau=\text { proj } \lim \tau_{\alpha}: \text { proj } \lim A_{\alpha} \rightarrow \text { proj } \lim A_{\alpha}
$$


is onto. We can verify directly that $\tau(x)=n x$ for all $x \in \operatorname{proj} \lim A_{\alpha}$. Therefore proj $\lim A_{\alpha}$ is divisible.

REMARK. For bounded Abelian groups compactness coincides with linear compactness. There is no gain of generality to assume that the $A_{\alpha}[n]$ are linearly compact instead of being compact.

COROLlARY 2. If $\left\{A_{\alpha}, \pi_{\alpha \beta}\right\}$ is an inverse system of Abelian groups where each $A_{\alpha}$ is a finite direct sum of quasi-cyclic groups then proj $\lim A_{\alpha}$ is divisible.

THEOREM 1. If $\left\{A_{\alpha}, \pi_{\alpha \beta}\right\}$ is an inverse system of Abelian groups where $A_{\alpha}$ are finite direct sums of quasi-cyclic and bounded Abelian groups, then

$$
A_{d}=\operatorname{proj} \lim \left(A_{\alpha}\right)_{d}, \quad A_{r}=\operatorname{proj} \lim \left(A_{\alpha}\right)_{r} .
$$

As a consequence $A$ is algebraically compact.

ProOF. We have an exact sequence of inverse systems of Abelian groups $0 \rightarrow\left\{\left(A_{\alpha}\right)_{d}, \pi_{\alpha \beta}^{\prime}\right\} \rightarrow\left\{A_{\alpha}, \pi_{\alpha \beta}\right\} \rightarrow\left\{\left(A_{\alpha}\right)_{r}, \pi_{\alpha \beta}^{\prime \prime}\right\} \rightarrow 0$ where $\pi_{\alpha \beta}^{\prime}, \pi_{\alpha \beta}^{\prime \prime}$ are homomorphisms induced by $\pi_{\alpha \beta}$. By Lemma 2 , the limit sequence

$$
0 \rightarrow \operatorname{proj} \lim \left(A_{\alpha}\right)_{d} \rightarrow \operatorname{proj} \lim A_{\alpha} \rightarrow \operatorname{proj} \lim \left(A_{\alpha}\right)_{r} \rightarrow 0
$$

is exact. By Corollary 2, proj $\lim \left(A_{\alpha}\right)_{d}$ is divisible. By [1, Proposition 39.4], proj $\lim \left(A_{\alpha}\right)_{r}$ is reduced. Therefore proj $\lim \left(A_{\alpha}\right)_{d}=A_{d}, \operatorname{proj} \lim \left(A_{\alpha}\right)_{r}=A_{r}$.

LEMMA 3. If $\left\{A_{\alpha}, \pi_{\alpha \beta}\right\}$ is an inverse system of torsion Abelian groups in which each $A_{\alpha}$ has only a finite number of nonzero primary components, then

$$
\text { proj } \lim A_{\alpha}=\prod_{p}\left(\operatorname{proj} \lim T_{p}\left(A_{\alpha}\right)\right) \text {. }
$$

This is a consequence of the universal property of inverse limit.

LEMMA 4. Let $A$ be an Abelian group. $A$ is the inverse limit of Abelian groups each of which is the direct sum of finite copies of $Z\left(p^{\infty}\right)$ iff $A=$ $\operatorname{Hom}_{Z}\left(B, Z\left(p^{\infty}\right)\right)$ where $B$ is a torsion-free Abelian group.

Proof. Let $\left\{A_{\alpha}, \pi_{\alpha \beta}\right\}$ be an inverse system of Abelian groups, where the $A_{\alpha}$ are finite direct sums of $Z\left(p^{\infty}\right)$, and $A=\operatorname{proj} \lim A_{\alpha}$.

Case I. All $\pi_{\alpha \beta}$ are onto. We have a direct system $\left\{\hat{A}_{\alpha}, \hat{\pi}_{\alpha \beta}\right\}$ of Abelian groups with

$$
\hat{A}_{\alpha}=\operatorname{Hom}_{Z}\left(A_{\alpha}, Z\left(p^{\infty}\right)\right), \quad \hat{\pi}_{\alpha \beta}=\operatorname{Hom}_{Z}\left(\pi_{\alpha \beta}, Z\left(p^{\infty}\right)\right) .
$$

Obviously $\hat{A}_{\alpha}$ are finite direct sums of $\hat{Z}_{p}$ and $\hat{\pi}_{\alpha \beta}$ are monomorphisms. We also have

$$
A_{\alpha}=\operatorname{Hom}_{Z}\left(\hat{A}_{\alpha}, Z\left(p^{\infty}\right)\right), \quad \pi_{\alpha \beta}=\operatorname{Hom}_{Z}\left(\hat{\pi}_{\alpha \beta}, Z\left(p^{\infty}\right)\right) .
$$


(These can be obtained either by direct computation or by Pontrjagin duality.) Therefore

proj $\lim A_{\alpha}=$ proj $\lim \operatorname{Hom}_{Z}\left(\hat{A}_{\alpha}, Z\left(p^{\infty}\right)\right)=\operatorname{Hom}_{Z}\left(\operatorname{inj} \lim \hat{A}_{\alpha}, Z\left(p^{\infty}\right)\right)$.

Since $\hat{A}_{\alpha}$ are torsion-free and $\hat{\pi}_{\alpha \beta}$ are $1-1, B=\operatorname{inj} \lim \hat{A}_{\alpha}$ is torsion-free.

Case II (the general case). Let $\pi_{\alpha}: A \rightarrow A_{\alpha}$ be the inverse limit projections, $A_{\alpha}^{\prime}=\operatorname{Im} \pi_{\alpha}$, and $\pi_{\alpha \beta}^{\prime}: A_{\beta}^{\prime} \rightarrow A_{\alpha}^{\prime}$ be induced by $\pi_{\alpha \beta}$, then $\left\{A_{\alpha}^{\prime}, \pi_{\alpha \beta}^{\prime}\right\}$ is an inverse system. Obviously the $\pi_{\alpha \beta}^{\prime}$ are onto, and $A=\operatorname{proj} \lim A_{\alpha}^{\prime}$. By Case $\mathrm{I}, A=\operatorname{Hom}_{Z}\left(B, Z\left(p^{\infty}\right)\right)$, where $B$ is a torsion-free Abelian group.

The converse is obvious.

COROLlaRY. An Abelian group is the inverse limit of finite direct sums of $Z\left(p^{\infty}\right)$ iff it is the direct product of copies of $Z\left(p^{\infty}\right)$ and copies of $Q$ where the number of $Q$ among the factors is either 0 or an infinite cardinal.

This is a consequence of [1, Theorem 47.1].

Combining all the previous results we have

THEOREM 2. An Abelian group $A$ is the inverse limit of Abelian groups each of which is a finite direct sum of quasi-cyclic and bounded Abelian groups iff the following conditions are satisfied:

(a) $A_{r}$ is complete.

(b) $A_{d}=\prod_{p} \operatorname{Hom}_{Z}\left(B_{p}, Z\left(p^{\infty}\right)\right)$, where the $B_{p}$ are torsion-free Abelian groups.

COROllary. Condition (b) can be replaced by

(b') $A_{d}$ is a direct product of quasi-cyclic groups and copies of $Q$ where the number of $Q$ among the factors is either 0 or an infinite cardinal.

Remark. Our results can be easily extended to modules over a Dedekind domain.

\section{REFERENCES}

1. L. Fuchs, Infinite abelian groups. Vol. 1, Pure and Appl. Math., vol. 36, Academic Press, New York, 1970. MR 41 \#333.

2. C. U. Jensen, On the vanishing of lim ${ }^{(t)}$, J. Algebra 15 (1970), 151-166. MR 41 \#5460.

3. A. Grothendieck, Eléments de géométrie algébrique. III. Etude cohomologique des faisceaux cohérents. I, Inst. Hautes Etudes Sci. Publ. Math. No. 11 (1961). MR 36 \#177c.

4. N. Bourbaki, Théorie des ensembles, Chap. I: Description de la mathématique formelle, Actualités Sci. Indust., no. 1212, Hermann, Paris, 1954; English transl., AddisonWesley, Reading, Mass., 1968. MR 16, 454; MR 38 \#5631.

Department of Mathematics, University of Iowa, Iowa City, Iowa 52240 